\title{
BMJ Open Effect of integrated urban and rural residents medical insurance on the utilisation of medical services by residents in China: a propensity score matching with difference-in-differences regression approach
}

Dai Su, ${ }^{1,2}$ Ying-chun Chen, ${ }^{1,2}$ Hong-xia Gao, ${ }^{1,2}$ Hao-miao Li, ${ }^{1,2}$ Jing-jing Chang, ${ }^{1,2}$ Di Jiang, ${ }^{1,2}$ Xiao-mei Hu, ${ }^{1,2}$ Shi-han Lei, ${ }^{1,2}$ Min Tan, ${ }^{1,2}$ Zhi-fang Chen ${ }^{1,2}$

To cite: Su D, Chen Y, Gao H, et al. Effect of integrated urban and rural residents medical insurance on the utilisation of medical services by residents in China: a propensity score matching with difference-in-differences regression approach. BMJ Open 2019;9:e026408. doi:10.1136/ bmjopen-2018-026408

- Prepublication history and additional material for this paper are available online. To view these files, please visit the journal online (http://dx.doi. org/10.1136/bmjopen-2018026408).

Received 1 September 2018 Revised 20 December 2018 Accepted 2 January 2019
Check for updates

(C) Author(s) (or their employer(s)) 2019. Re-use permitted under CC BY-NC. No commercial re-use. See rights and permissions. Published by BMJ.

For numbered affiliations see end of article.

Correspondence to Dr Ying-chun Chen; chenyingchunhust@163.com

\section{ABSTRACT}

Objectives In this study, we aim to evaluate the effect of urban and rural resident medical insurance scheme (URRMI) on the utilisation of medical services by urban and rural residents in the four pilot provinces.

Setting and participants The sample used in this study is 13305 individuals, including 2620 in the treatment group and 10685 in the control group, from the 2011 and 2015 surveys of China Health and Retirement Longitudinal Study.

Outcome measures Propensity score matching and difference-in-differences regression approach (PSM-DID) is used in the study. First, we match the baseline data by using kernel matching. Then, the average treatment effect of the four outcome variables are analysed by using the DID model. Finally, the robustness of the PSM-DID estimation is tested by simple model and radius matching. Results Kernel matching have improved the overall balance after matching. The URRMI policy has significantly reduced the need-but-not outpatient care and significantly increased outpatient care cost and inpatient care cost for rural residents, with DID value of $-0.271,0.090$ and 0.256 , respectively. After robustness test, the DID competing results of four outcome variables are consistent.

Conclusions URRMI has a limited effect on the utilisation of medical and health services by all residents, but the effect on rural residents is obvious. The government should establish a unified or income-matching payment standard to prevent, control the use of medical insurance funds and increase its efforts to implement URRMI integration in more regions to improve overall fundraising levels.

\section{INTRODUCTION}

In 2005, the WHO urged member states to commit to universal health coverage (UHC) to ensure that all people have access to the health services they need and that these member states do not create economic risks or poverty in health service utilisation. Member

\section{Strengths and limitations of this study}

- This study used the propensity score matching and difference-in-differences regression approach method to evaluate the effect of urban and rural resident medical insurance scheme (URRMI) integration on the utilisation of medical services by residents in China, which has not been analysed before.

- The data are derived from China Health and Retirement Longitudinal Study and thus can be regarded credible. We analysed the effect of pilot provinces that implemented the URRMI policy. The findings can provide a rich experience for the URRMI policy to be promoted nationwide.

- The unobservable characteristics of individual level could not be addressed in the analysis model of this study.

- The utilisation of medical services by urban and rural residents under 45 years old cannot be considered, which limits the scope of application of this conclusion.

states need to strengthen their political will to realise fairness and respect for the right to health of all people. ${ }^{1}$ WHO has recommended three aspects that should be considered for achieving UHC: population coverage (broadness), service coverage (depth) and health cost coverage (height). Governments around the world are actively taking action to respond to WHO's goal of global health coverage. ${ }^{2}$ Some developing countries and low-income countries, especially emerging economies such as China, Thailand, South Africa and Mexico, have implemented social health insurance (SHI) reform programmes as the first step of UHC. ${ }^{3}$ 
In 2009, the Chinese government launched a new round of national healthcare system reform to provide healthcare services that are accessible and fair to residents. ${ }^{4}$ With the continuous implementation of the reform, the Chinese government hopes to reduce the economic burden of residents and improve the utilisation level of medical services. The health outcomes can be achieved by establishing SHI with multilevel and extensive coverage, thereby promoting the realisation of UHC. ${ }^{5}$ The current SHI system in China has an obvious dual structure of urban-rural areas, consists of the urban employee basic medical insurance (UEBMI), the urban resident basic medical insurance (URBMI) schemes and the new rural cooperative medical system (NRCMS). ${ }^{6}$ By the end of 2015, the number of individuals participating in SHI reached 1.34billion in China (coverage reached $97.8 \%){ }^{7}$

However, major differences exist in the coverage, fund raising and operation of UEBMI, URBMI and NRCMS. UEBMI was piloted in the 'Two Rivers' region of China in 1994 and was officially implemented in 1998. UEBMI is mandatory for all urban employees and retired citizens, and it covered 295 million individuals in $2016 .{ }^{8}$ The premium for UEBMI ( $8 \%$ of the salary) is paid by employers $(6 \%$ as tax) and employees $(2 \%)$ on a monthly basis. Retirees do not pay premiums, and this premium is shouldered by employees. The funds of SHI consist of pooling funds and personal accounts. All personal payments and $30 \%$ of the employer's payment of UEBMI are included in a personal account that is used for outpatient expenses. The rest of the payments are included in a pooling fund allocated for inpatient reimbursements. URBMI was piloted in 88 cities in China in 2007 and was fully implemented in 2010. URBMI mainly covers students, children and non-employed urban residents and adheres to the principle of voluntary participation. By the end of 2016, URBMI has covered 453 million individuals, and both UEBMI and URBMI have covered more than $90 \%$ of urban population in China. The financing of URBMI mainly comes from financial subsidies and individual payments. In 2015, the per capita fundraising amount of URBMI was approximately $¥ 515$ (US\$1=¥6.90) and personal payment accounted for $21.75 \%$. $^{9}$ Piloted in 2003 and fully implemented in 2007, NRCMS covers all rural residents, and it is funded by individuals, collectives and governments. NRCMS also involves the voluntary participation of families. In 2015, NRCMS covered 670 million individuals (coverage of $98.80 \%$ ). The average fundraising was $¥ 490$, and individual payments accounted for $19.25 \% .^{8}$ In URBMI and NRCMS, premiums are paid on a yearly basis, and the establishment of pooling funds (mainly funded by the government) compensates hospitalisation. Some areas have also attempted to compensate outpatient costs. In 2013, the reimbursement rates of UEBMI, URBMI and NRCMS were approximately $85 \%$, $60 \%$ and $50 \%$, respectively. The compensation levels of URBMI and NRCMS are much lower than that of UEBMI. $^{10-12}$
The remarkable progress in establishing a comprehensive SHI system is rapid and impressive in China. The broad coverage of the SHI system has improved access to health services and promoted utilisation of outpatient and inpatient financing, thereby reducing the proportion of out-of-pocket (OOP) costs and improving the health of residents. ${ }^{13}$ However, the scattered and gradual development of the three basic medical insurances has formed a broken SHI that cannot meet the dynamic health service needs of different population groups, thus resulting in structural defects in the utilisation of medical services. ${ }^{14}$ First, as urbanisation accelerates in China, population migration between urban and rural areas has become common. Multiple insurance coverages are repeated, resulting in inefficient use of medical insurance funds. Moral hazards and adverse selection are also not effectively managed. Second, due to the large difference in the compensation scope and levels between different medical insurances, personal and public financial burdens have become aggravated. Thus, resident participation in different basic insurance plans in favour of the fair use of medical services cannot be guaranteed. This funding gap affects societal health outcomes. ${ }^{15}$ To improve the fairness, sustainability and efficiency of URBMI and NRCMS and to promote the process of UHC, the Chinese government piloted the integration of URBMI and NRCMS in some areas in 2008, which can be essentially described as the pooling funds of urban and rural resident medical insurance scheme (URRMI) at the province level. URRMI has two main forms. In most areas, the 'one system, two standards' type is implemented, where two standards of payment and compensation are set, and residents are free to choose any standard. The second type is 'one system, one standard', which is implemented in economically developed areas, which demonstrates a clear unification of payment standards and compensation standards. In 2016, the Chinese government issued the 'Guiding Opinions on Integrating the Urban and Rural Residents Basic Medical Insurance System', requiring that the URRMI must be unified in terms of coverage, financing policies, treatment, medical insurance catalogues, the management of designated hospitals and fund. ${ }^{15}$ The provinces introduced the implementation plan before the end of the year and the nationwide launch in 2019, the core of which is fair financing and equal benefits. ${ }^{16}$

Chinese scholars have begun to explore the impact of URRMI on the utilisation of medical services by residents since the piloting of URRMI. Gu et $a l^{17}$ decomposed differences in the utilisation of medical services between urban and rural areas in the URRMI integration area. Findings showed that the differences in descriptive statistics may have underestimated the unfairness of the utilisation of medical services between urban and rural areas. The main factors that have caused the difference in the utilisation of urban and rural medical services are 
income and healthcare policy. Ma et $a l^{18}$ reported that URRMI has significantly increased the frequency and cost of outpatient service utilisation for rural residents, but no evidence has been established that it significantly affects hospitalisation. $\mathrm{Liu}^{19}$ argued that URRMI does not have a substantial impact on the level of medical service utilisation in China. The reason may be that URRMI has not significantly narrowed the urban-rural difference in the actual compensation rate. Huang and Zhang ${ }^{20}$ analysed the 1600 insured rural residents in Guangzhou and found that their utilisation of medical services has improved significantly, but this is not the case for urban residents. The total medical expense of rural residents during the year is $54.9 \%$ lower than that of urban residents. The number of outpatient visits and inpatient visits of rural residents during the year was 0.74 times and 0.15 times lower than that of urban residents, respectively. The proportion of OOP cost for medical services by urban and rural residents remains high at nearly $50 \%$, but the proportion for rural residents is approximately $8.7 \%$ higher than that of urban residents.

Existing research has its shortcomings in terms of quantitative evaluation. First, few control variables are used, and the influence of confounding factors on the implementation of policies cannot be accurately excluded. Second, the attention given to the effect of policy implementation is insufficient, and the number of research references is limited. The conclusions presented by extant research are also inconsistent. Finally, most current research focus on utilisation of medical services by rural residents, but studies on the utilisation of urban and rural health services and their differences are insufficient.

In this study, we use the large database of China Health and Retirement Longitudinal Study (CHARLS) for analytical support and the propensity score matching and difference-in-differences regression approach (PSMDID) method-a complete established method in health service research-to assess the effect of URRMI on the utilisation of medical services by urban and rural residents. ${ }^{21-23}$ Thus, we attempt to provide suggestions on the practicality of the policy and offer an important reference for the full implementation of the URRMI system in China.

\section{METHODS}

\section{Data source/sample}

The data used in this study are derived from the 2011 baseline survey and the 2015 tracking survey of CHARLS. The CHARLS survey collects a set of high-quality microdata representing families and individuals aged 45 years and older in China to analyse the ageing Chinese population and promote interdisciplinary research on ageing. The CHARLS questionnaire includes demographic backgrounds, family structure and financial support, health status and functioning, healthcare and insurance, work, retirement and pension, income, expenditures and assets, and housing characteristics. The questionnaire design draws on international experience, including the Health and Retirement Study, the English Longitudinal Study of Aging and the Survey of Health, Aging and Retirement in Europe. The project adopted multistage sampling, and probability proportionate to size sampling methods were adopted in the county/district and village sampling stages. CHARLS pioneered the electronic drawing software (CHARLS-GIS) technology and used the map method to create village-level sampling frames.

The CHARLS baseline survey was launched in 2011 and covered 150 county-level units, 450 village-level units and 17708 individuals from 10257 households in 28 provinces, municipalities and autonomous regions. In general, the CHARLS baseline is a good representation of the elderly population of China. Overall response rate was $80.51 \%$, of which the rural response rate was as high at $94.15 \%$. Sampling are conducted every 2 years. By 2015 , the sample had covered 21069 individuals from 12083 households, with an overall response rate of $82.63 \%$, of which the rural response rate was $91.74 \%$.

In this study, 31777 observations were admitted from CHARLS database between 2011 and 2015. After selection, the remaining 26610 observations were separated into URRMI group (treatment group) and non-URRMI group (control group) (figure 1). This study selected the provinces that piloted the URRMI in 2011-2015 as the treatment group, includes Zhejiang, Shandong, Guangdong and Chongqing. Otherwise, as the control group. This ensures that there is no pilot province for URRMI before 2011, and there are some pilot provinces after 2015. The final populations used in this paper is 13305 people, including 2620 in the treatment group and 10685 in the control group.

\section{Variables}

For this study, two types of outcome variable are considered in our analysis: (1) frequency of healthcare service utilisation, including need-but-not outpatient care (NOC) and need-but-not inpatient care (NIC) and (2) healthcare service cost, including outpatient care cost (OCC) and inpatient care cost (ICC). The URRMI is set as the treatment variable. Based on the framework of the Anderson Health Services Utilization Initial Model, ${ }^{24}$ the elements that ultimately result in the utilisation of health services include propensity characteristics, capacity resources and health needs. We synthesise the structure of the CHARLS questionnaire and screen the explanatory variables contained in the three elements as follows: (1) propensity characteristics: gender, age, hukou status, marital status and education level; (2) capacity resources: family income, receive child, given child, live with child, pension and retirement; and (3) health needs: health status, chronic, smoke, drink, activities of daily living, cognition and depression. The OCC and ICC were natural logarithm transformed as the original data are skewed to the right. The calculation and definition of the outcome 


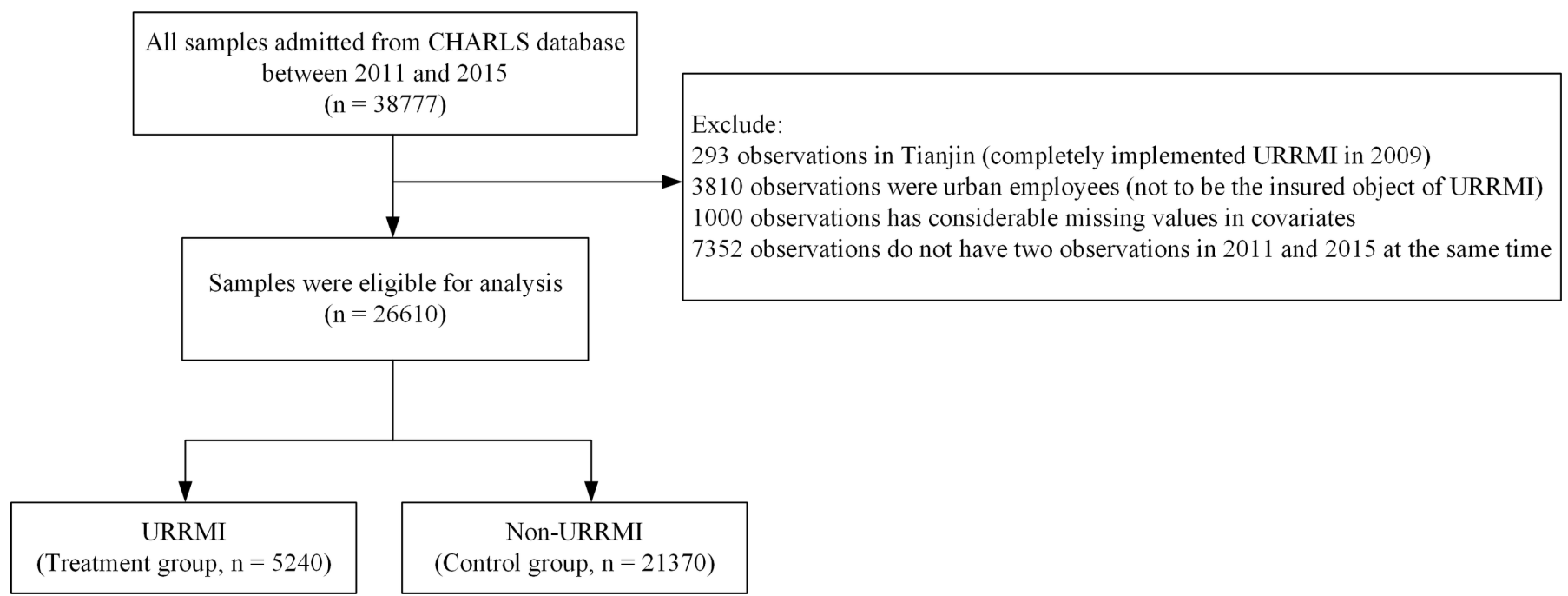

Figure 1 Study design and flow chart of the observations selection and the classify of those observations with and without URRMI for propensity score matching. The $\mathrm{n}$ stands for the observations between 2011 and 2015, respectively. CHARLS, China Health and Retirement Longitudinal Study; URRMI, urban and rural residents medical insurance.

and explanatory variables is shown in online supplementary table 1 .

\section{Statistical analysis}

Propensity score matching

To estimate the effects of URRMI integration on the utilisation of medical services by residents in China, the outcomes between treatment group and comparable control group are compared. Although the treatment group can be easily selected from the CHARLS database, some characteristics of the control group may be inconsistent with those of the treatment group. To address this shortcoming, we constructed an appropriate control group by using PSM. The PSM method is widely used in estimating the effects of health and other policy interventions, whereas randomised controlled trials are not feasible. ${ }^{25}$ Matching, which is based on propensity scores, can identify individuals in the control group with similar characteristics as those affected by the policy.

Before estimating the propensity scores based on a rich set of covariates from logistic regression, we initially restricted the potential control group by considering the key individual characteristics of the treatment group and the availability of data prior policy implementation. To test the balancing property of each observed covariate and the overall balance between the treatment and control groups in the baseline and to verify the reduced sampling bias achieved through matching, kernel matching was used for our main analysis. We used a with replacement kernel matching at the bandwidth of 0.06 , and the kernel type is epan kernel. The kernel types and bandwidths are frequently mentioned in the literature. ${ }^{26-28}$ Observations not on the common support were excluded from the analysis. We checked the balance of means of covariates after matching by examining the standardised mean differences between the control group and the treatment group in the baseline (ie, the baseline is also checked before and after matching). After matching, the bias should be $\leq 5 \%$ (or $\mathrm{p}$ value $>0.1$ ) to establish adequate matching. Then, we calculated the achieved percentage of the bias reduction and examined each covariate's standardised bias percentage before and after matching. Finally, we determined whether the kernel matching is appropriate based on the mean reduction bias and median reduction bias of overall balance. ${ }^{29}$

\section{DID model}

The most important premise of the DID model is that the treatment group and the control group must meet the common-trend assumption. ${ }^{30}$ If the URRMI policy has not been implemented, then no systematic differences can be observed in the trends of medical service utilisation over time between individuals who participated and did not participate in the URRMI. In reality though, the common-trend assumption cannot be easily satisfied. We therefore used the samples after conducting a panel balance for waves 2011 and 2015.

The following equation of DID model is estimated for continuous outcomes:

$$
\mathrm{Y}_{i t}=\begin{aligned}
& \beta_{1}+\beta_{2} \text { URRMI }_{i}+\beta_{3} \text { After }_{t}+\delta\left(\text { URRMI }_{i}\right. \\
& \\
& \times A f t e r_{t}+\gamma \times X_{i t}+\lambda_{j}+\eta_{j}+\varepsilon_{i t}
\end{aligned}
$$

The following equation of DID model is estimated for binary outcomes:

$$
\operatorname{logit}\left(Y_{i t}\right)=\begin{aligned}
& \beta_{1}+\beta_{2} \text { URRMI }_{i}+\beta_{3} \text { After }_{t}+\delta\left(\text { URRMI }_{i}\right. \\
& \\
& \times A f t r_{t}+\gamma \times X_{i t}+\lambda_{j}+\eta_{j}+\varepsilon_{i t}
\end{aligned}
$$

Where $Y_{i t}$ stands for healthcare service utilisation of resident at time $t$; the treatment variable $U R R M I_{i}$ is a binary indicator; it represents the group dummy variable. $U R R M I_{i}=1$ represents the treatment group that the individuals come from the province that have been implemented the URRMI; URRMI $I_{i}=0$ represents the control group that the individuals come from the province that 
have not begun to implement the URRMI; After ${ }_{t}$ represents the time dummy variable. After ${ }_{t}=0$ means the time before the URRMI implemented (the year of 2011), and After $_{t}=1$ means the time after the URRMI implemented (the year of 2015); the variable $U R R M I_{i} \times$ After $_{t}$ denotes the interaction between groups and time; $X_{i t}$ represents a set of individual covariates of resident $i$ at time $t ; \lambda_{j}$, $\eta_{j}$ and $\varepsilon_{i t}$ denote three error terms and contains information other than the main variables of the model. For the purpose of nullifying the roles of province and community level unobserved characteristics, $\lambda_{j}$ and $\eta_{j}$ represent province and community level fixed effects, respectively; $\varepsilon_{i t}$ means an independently distributed error term that represents individual level and other unobservable characteristics. $\delta$ is calculated by DID model and denotes average treatment effect of the policy; we called $\delta$ the DID value in this paper.

To estimate the effects of URRMI integration on the utilisation of medical services by residents in China, we used a PSM-DID regression approach rather than a matching estimator alone to estimate the policy effect. These two methods are complementary to one another and can be applied for causal inferences to counter any selection bias or confounding. ${ }^{31}$ The combination of methods to counter biases and the confounding of different sources, and the comparison of results, are encouraged in the literature. An advantage of the PSM-DID approach is its capability to handle relatively weak assumptions, which is not the case for matching estimators, thus helping DID meeting common trend on observables. ${ }^{22}$ Therefore, to completely eliminate biases in the sampled selection, controlling all covariates that may affect the selection and results is necessary, but this matching technique often requires as many control variables as possible. To examine robustness of the DID results after PSM, we presented DID competing results coming from fixed-effect model and simple model, one matching method versus another matching method. Table 1 presents an overview of the characteristics of our study sample that has been stratified by treatment status. Stata 14.0 in Windows was used for

Table 1 Characteristics of study sample in the CHARLS 2011 and 2015

\begin{tabular}{|c|c|c|c|c|c|c|c|c|}
\hline \multirow[b]{4}{*}{ Variable } & \multicolumn{4}{|l|}{2011} & \multicolumn{4}{|l|}{2015} \\
\hline & \multicolumn{2}{|l|}{ Treatment } & \multicolumn{2}{|l|}{ Control } & \multicolumn{2}{|l|}{ Treatment } & \multicolumn{2}{|l|}{ Control } \\
\hline & \multicolumn{2}{|l|}{$(n=2620)$} & \multicolumn{2}{|l|}{$(n=10685)$} & \multicolumn{2}{|l|}{$(n=2620)$} & \multicolumn{2}{|l|}{$(n=10685)$} \\
\hline & Mean & SD & Mean & SD & Mean & SD & Mean & SD \\
\hline NOC & 0.21 & 0.41 & 0.30 & 0.46 & 0.28 & 0.45 & 0.34 & 0.47 \\
\hline OCC & 4.97 & 1.75 & 5.04 & 1.62 & 5.60 & 1.82 & 5.69 & 1.70 \\
\hline NIC & 0.06 & 0.24 & 0.09 & 0.29 & 0.11 & 0.32 & 0.13 & 0.34 \\
\hline ICC & 8.25 & 1.34 & 7.78 & 1.37 & 8.51 & 1.35 & 8.26 & 1.44 \\
\hline Gender & 0.53 & 0.50 & 0.54 & 0.50 & 0.53 & 0.50 & 0.54 & 0.50 \\
\hline Age & 58.87 & 10.02 & 58.41 & 9.52 & 62.50 & 9.75 & 62.14 & 9.39 \\
\hline Hukou status & 0.12 & 0.33 & 0.14 & 0.34 & 0.18 & 0.43 & 0.17 & 0.40 \\
\hline Marital status & 0.12 & 0.33 & 0.12 & 0.32 & 0.16 & 0.36 & 0.16 & 0.36 \\
\hline Education level & 3.15 & 1.85 & 3.15 & 1.87 & 3.15 & 1.85 & 3.12 & 1.87 \\
\hline Family income & 18877.82 & 35510.49 & 15409.88 & 31934.90 & 18523.48 & 63822.28 & 12681.72 & 84475.73 \\
\hline Health status & 2.65 & 1.03 & 2.44 & 1.00 & 3.30 & 1.07 & 3.51 & 1.04 \\
\hline Chronic & 1.06 & 1.23 & 1.41 & 1.40 & 0.37 & 0.70 & 0.42 & 0.76 \\
\hline Smoke & 0.28 & 0.45 & 0.28 & 0.45 & 0.39 & 0.49 & 0.39 & 0.49 \\
\hline Drink & 0.34 & 0.48 & 0.32 & 0.47 & 0.35 & 0.48 & 0.32 & 0.47 \\
\hline$A D L$ & 75.64 & 6.83 & 75.14 & 6.88 & 73.31 & 9.89 & 72.42 & 10.07 \\
\hline Cognition & 5.25 & 2.32 & 5.02 & 2.27 & 5.62 & 1.77 & 5.34 & 1.78 \\
\hline Depression & 29.46 & 10.50 & 28.49 & 10.26 & 27.45 & 11.68 & 26.79 & 11.12 \\
\hline Receive child & 998.06 & 8009.74 & 716.45 & 3816.16 & 4039.64 & 9800.35 & 4083.56 & 10124.27 \\
\hline Given child & 537.11 & 6786.44 & 471.67 & 6634.05 & 4365.37 & 56796.50 & 3851.11 & 20546.07 \\
\hline Live with child & 0.44 & 0.50 & 0.36 & 0.48 & 0.46 & 0.50 & 0.49 & 0.50 \\
\hline Pension & 444.23 & 2290.80 & 314.75 & 2274.78 & 2663.95 & 8009.51 & 2043.58 & 7026.76 \\
\hline Retirement & 0.92 & 0.27 & 0.90 & 0.30 & 0.92 & 0.28 & 0.90 & 0.30 \\
\hline
\end{tabular}

The mean shows the annual average for each variable.

$A D L$, activities of daily living; ICC, inpatient care cost; NIC, need-but-not inpatient care; NOC, need-but-not outpatient care; OCC, outpatient care cost. 
Table 2 Test the overall balance from kernel matching

\begin{tabular}{|c|c|c|c|c|c|}
\hline & Pseudo $\mathbf{R}^{2}$ & $\operatorname{LR} \chi^{2}$ & $\mathrm{P}>\chi^{2}$ & Mean bias & Median bias \\
\hline Sample & (1) & (2) & (3) & (4) & (5) \\
\hline \multicolumn{6}{|l|}{ NOC } \\
\hline Raw sample before matching & 0.034 & 73.69 & 0.000 & 10.5 & 10.8 \\
\hline Matched sample after kernel matching & 0.001 & 0.79 & 1.000 & 1.3 & 1.0 \\
\hline \multicolumn{6}{|l|}{ OCC } \\
\hline Raw sample before matching & 0.033 & 73.91 & 0.000 & 10.4 & 10.1 \\
\hline Matched sample after kernel matching & 0.001 & 0.92 & 1.000 & 1.4 & 1.3 \\
\hline \multicolumn{6}{|l|}{$\mathrm{NIC}$} \\
\hline Raw sample before matching & 0.034 & 73.69 & 0.000 & 10.5 & 10.8 \\
\hline Matched sample after kernel matching & 0.001 & 0.79 & 1.000 & 1.3 & 1.0 \\
\hline \multicolumn{6}{|l|}{ ICC } \\
\hline Raw sample before matching & 0.151 & 43.09 & 0.001 & 20.0 & 18.3 \\
\hline Matched sample after kernel matching & 0.015 & 1.85 & 1.000 & 3.0 & 2.9 \\
\hline
\end{tabular}

All results are computed using the Stata module of psmatch2.

ICC, inpatient care cost; LR, likelihood ratio; NIC, need-but-not inpatient care; NOC, need-but-not outpatient care; OCC, outpatient care cost.

statistical analysis. The two-sided statistical significance level was set to 0.05 .

\section{Patient and public involvement}

All data in this study were derived from the CHARLS database, so no patients and the public were involved in the study design, the outcome measures, data analysis or interpretation of the results. Results will be disseminated to study participants via this publication. The study samples are thanked in the acknowledgements.

\section{RESULTS}

\section{Balancing proper test for PSM result}

We examined the balancing property of each observed covariate between the treatment and control groups and the reduced sampling bias achieved through kernel matching. The results suggest that almost all observable covariates are sufficiently balanced by the matching between the treatment and control groups. The initial differences in the two groups are reduced considerably and have become statistically insignificant at $5 \%$ (online supplementary table 2).

As shown by table 2, the overall balancing properties can be verified by comparing the joint significance of all matching variables in the logit models before and after matching. In column 1 , the pseudo $\mathrm{R}^{2}$ of results after PSM by using kernel matching are much lower for the matched sample than for the raw sample. In columns 2 and 3, the matching covariates are jointly significant before matching and became insignificant after matching ( $p$ value $>0.05$ ). The results further indicate that kernel matching have improved the overall balance after matching. Columns 4 and 5 consistently show that both the mean and the median of the absolute standardised bias have been reduced substantially by kernel matching.
Effects of URRMI on the utilisation of medical services by residents

The effect of URRMI on the utilisation of medical services by residents was estimated on the basis of kernel matching. We examined the impact of URRMI integration on NOC, OCC, NIC and ICC. The sample was divided into urban and rural residents, which is the most sensitive variable to the characteristics of the population in the URRMI reform. Then, DID analysis is conducted for the three types of sampling, namely, all samples, rural samples and urban samples (table 3 and table 4).

From the analytical results of all the samples, no significant difference exists in the utilisation of medical services between urban and rural residents before and after the implementation of URRMI. In the sample of rural residents, the URRMI policy significantly reduced the value of NOC, and increased the value of OCC and ICC, with PSM-DID values of $-0.271,0.090$ and 0.256 , respectively.

\section{Testing the robustness of PSM-DID estimation}

To ensure the robustness of the PSM-DID results, we further test DID competing results coming from fixed-effect model and simple model, one matching method versus another matching method. First, we performed linear and logistic regression using a simple model of DID without considering the province and community fixed effects. The results were consistent with the PSM-DID results in tables 3 and 4 (online supplementary table 3-4). Then, we used the radius matching method to match the original panel data between treatment and control group in the baseline. Linear and logistic regression containing provincial and community fixed effects were performed, and we found that the results were also consistent with the previous PSM-DID results in tables 3 and 4 (online supplementary table 5-6). It indicated that although 
Table 3 Effect of URRMI on the frequency of healthcare service utilisation for residents (NOC and NIC): PSM-DID estimate result

\begin{tabular}{|c|c|c|c|c|c|c|}
\hline \multirow[b]{2}{*}{ Variables } & \multicolumn{3}{|l|}{ NOC } & \multicolumn{3}{|l|}{ NIC } \\
\hline & All & Urban & Rural & All & Urban & Rural \\
\hline URRMI×After & $-0.191(0.151)$ & $-0.185(0.191)$ & $-0.271^{\star \star}(0.247)$ & $0.165(0.240)$ & $-0.139(0.411)$ & $0.320(0.302)$ \\
\hline After & $0.029(0.086)$ & $-0.111(0.149)$ & $0.092(0.107)$ & $0.668^{\star \star \star}(0.129)$ & $0.662^{\star * \star}(0.232)$ & $0.672^{\star \star *}(0.156)$ \\
\hline Covariates & Yes & Yes & Yes & Yes & Yes & Yes \\
\hline $\begin{array}{l}\text { Community-level } \\
\text { fixed effects }\end{array}$ & Yes & Yes & Yes & Yes & Yes & Yes \\
\hline Constant & $0.087(0.524)$ & $0.387(0.917)$ & $0.066(0.694)$ & $0.799(0.693)$ & $1.396(1.198)$ & $0.228(0.924)$ \\
\hline $\mathrm{R}^{2} /$ pseudo $\mathrm{R}^{2}$ & 0.022 & 0.030 & 0.025 & 0.063 & 0.073 & 0.063 \\
\hline
\end{tabular}

Robust SEs in parentheses.

Constant represents beta-1, 'URRMI' represents beta-2, 'After' represents beta-3 and 'URRMIxAfter' represents delta (DID value) in equation (2).

Coefficient value and SEs are estimated by logistic regression.

${ }^{* \star *} \mathrm{P}<0.01 ;{ }^{* \star} \mathrm{p}<0.05 ;{ }^{*} \mathrm{p}<0.1$.

NIC, need-but-not inpatient care; NOC, need-but-not outpatient care; PSM-DID, propensity score matching and difference-indifferences regression approach; URRMI, urban and rural resident medical insurance.

there are inevitable deviations in coefficient value and SEs through the results of the robustness test, this does not affect the robustness of the conclusions in this paper.

\section{DISCUSSION}

Our study found that, as the year of 2015, the URRMI policy has not significantly affected the utilisation of medical services by all residents in the four pilot provinces, as depicted by the NOC (DID value $=-0.191$, $\mathrm{p}$ value $=0.756)$, OCC $(\mathrm{DID}$ value $=0.075$, $\mathrm{p}$ value $=0.603)$, NIC (DID value $=0.165, p$ value $=0.408$ ) and ICC (DID value $=0.110$, p value $=0.805$ ). There result can be attributed to two reasons: first, the implementation of URRMI may have not led to significant changes in the overall actual compensation rate. Evidence from developing countries, such as Brazil and Mexico, have shown that the

Table 4 Effect of URRMI on the cost of healthcare service utilisation for residents (OCC and ICC): PSM-DID estimate result

\begin{tabular}{|c|c|c|c|c|c|c|}
\hline \multirow[b]{2}{*}{ Variables } & \multicolumn{3}{|l|}{ OCC } & \multicolumn{3}{|l|}{ ICC } \\
\hline & All & Urban & Rural & All & Urban & Rural \\
\hline URRMI×After & $0.075(0.027)$ & $0.058(0.022)$ & $0.090^{\star *}(0.066)$ & $0.110(0.054)$ & $-0.144(0.040)$ & $0.256^{\star \star}(0.047)$ \\
\hline After & $0.647^{\star \star *}(0.076)$ & $0.516^{\star \star \star}(0.131)$ & $0.680^{\star \star \star}(0.093)$ & $0.154(0.091)$ & $0.187(0.051)$ & $0.139(0.013)$ \\
\hline Covariaetes & Yes & Yes & Yes & Yes & Yes & Yes \\
\hline $\begin{array}{l}\text { Community-level fixed } \\
\text { effects }\end{array}$ & Yes & Yes & Yes & Yes & Yes & Yes \\
\hline Constant & $5.427^{\star \star \star}(0.500)$ & $5.652^{\star \star \star}(0.966)$ & $5.017(0.636)$ & $9.140^{\star \star \star}(0.510)$ & $9.652^{\star \star \star}(0.795)$ & 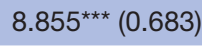 \\
\hline$R^{2} /$ pseudo $R^{2}$ & 0.103 & 0.125 & 0.103 & 0.105 & 0.094 & 0.122 \\
\hline Number of observations & 4551 & 1406 & 3145 & 2446 & 835 & 1611 \\
\hline
\end{tabular}

Robust SEs in parentheses.

Constant represents beta-1, 'URRMI' represents beta-2, 'After' represents beta-3 and 'URRMIxAfter' represents delta (DID value) in equation (1).

Coefficient value and SEs are estimated by linear regression.

${ }^{* * *} \mathrm{P}<0.01 ;{ }^{* *} \mathrm{p}<0.05 ;{ }^{*} \mathrm{p}<0.1$.

ICC, inpatient care cost; OCC, outpatient care cost. 
'one system, two standards' model for universal unified medical insurance can fully consider the actual gap in the income levels between urban and rural residents. In particular, this model construction scheme can reflect the fairness of the grade choice of urban and rural residents. ${ }^{32}$ This model is also applicable for China. The 'one system, two standards' model is a transitional measure in China's reform health programme to guarantee insured residents in the pilot areas. This practice allows insured residents to choose the payment standard that suits their payment capability, and it gives rural residents the right to choose a payment standard independently. ${ }^{15} 3334$ This model also gives rural residents the right to choose highgrade medical insurance that only urban residents used to enjoy. However, although it eliminates discrimination based on identity and household registration, the model cannot completely eliminate the difference in fundraising and forms of treatment within the system. ${ }^{35}$ In the pilot areas of China, majority of the agricultural hukou residents with low income and low cultural level have opted to retain their original payment habits. These residents may not have the strong willingness to participate in second-grade URRMI (ie, the compensation ratio is relatively high); In this study, no significant change has been observed in the utilisation of medical services, which is consistent with the findings of Gu et al ${ }^{17}$ and Liu. ${ }^{19}$ Therefore, to further promote URRMI integration, particularly as the income gap between urban and rural residents gradually narrows, the 'one system, one standard' may be achieved by setting a unified payment standard and exploring a suitable financing mechanism linked with the per capita disposable income of urban and rural residents. As such, urban and rural residents can equally improve the utilisation of medical services. ${ }^{36-38}$ Second, URRMI follows the principle in which the treatment level of postintegration refers to a 'higher treatment level' compared with the preintegration of URBMI and NRCMS, thus achieving a smooth transition for the reimbursement policy of URBMI. The treatment level of the original policy, including outpatient care, hospitalisation compensation and serious illness insurance, has generally remained unchanged. Therefore, this study was unable to establish a significant impact on the level of health service utilisation of urban residents. A previous study has suggested that the compensation level of URRMI be gradually increased to reduce catastrophic health expenditure. $^{39}$

After the implementation of URRMI, the ICC of rural residents have significantly improved (DID value $=0.256$, $\mathrm{p}$ value $=0.032$ ), which is consistent with previous research. ${ }^{40-42}$ Considering the necessity of hospitalisation needs and the design of NRCMS that is centres on hospitalisation compensation, the rural residents might have mainly considered their own disease severity and hospital service level in selecting inpatient hospitals. The rural residents also have relatively low sensitivity towards compensation level. On the basis of hospital distribution and rural inpatient in recent years, and despite the emphasis on initial medical care through the community and healthcare grading system, rural residents may have preferred high-level hospitals with better service capabilities over primary medical hospitals. In addition, after URRMI integration, the treatment levels in the pilot areas have markedly improved alongside the expanded scope of hospitals designated for medical insurance, the timely settlement of medical services with the use of social security identification and the reduced medical expenses of residents. Similarly, the utilisation of medical services by insured rural residents can be improved and high hospitalisation costs can be resolved. Therefore, preventing the use and controlling the risk of medical insurance funds requires sufficient attention.

However, the effect of URRMI on the hospitalisation of rural residents should not be the main basis for evaluating policy performance, as the indicators of outpatient service utilisation can also reflect the current effect of URRMI on the level of medical service utilisation of rural residents. After integration, the NOC of rural residents (DID value $=-0.271, \mathrm{p}$ value $=0.043$ ) significantly reduced and OCC (DID value $=0.090, \mathrm{p}$ value $=0.026$ ) significantly increased, and the change is mainly due to the policies on outpatient pooling funds and outpatient chronic disease, which have been prioritised by URBMI and NRCMS before URRMI implementation in the pilot areas. In terms of the outpatient pooling fund policy, the annual maximum payment limit of NRCMS is slightly higher than that of URBMI. However, in terms of the outpatient chronic disease policy, the number of special chronic diseases in NRCMS is significantly larger than that in URBMI, and the reimbursement rate of NRCMS is higher, but the annual maximum payment limit is lower, than URBMI. ${ }^{43}$ On the basis of the principle of 'treatment level of postintegration' (ie, higher treatment level at postintegration compared with preintegration for URBMI and NRCMS), the scope of disease and the annual maximum payment limit in the outpatient pooling funds and outpatient chronic disease policy has improved, and this phenomenon may have greatly stimulated the health service utilisation of rural residents. The reasons may be related to the public health and family doctor enlistment services of rural primary medical hospitals, the policies of which are much more publicised to rural residents. Meanwhile, the utilisation level of outpatient services by urban residents might have been affected by multiple factors, such as the time lag effect and relatively high economic conditions, such that the current level of utilisation of medical services remains unrecognisable. Therefore, the government should increase efforts to implement URRMI integration in more regions, raise the level of overall pooling funds and strengthen policy propaganda to further promote social substantive equity. ${ }^{445}$

\section{LIMITATIONS}

This study has several limitations. First, an important assumption in the implementation of PSM-DID is that the 
model should contain all covariates that may influence the policy effect before and after matching. Unobservable covariates will cause different trends between the treatment group and the control group, and such an instance may have led to biased results in this study. Second, in the PSM process, kernel matching and radius matching were selected for the evaluation of the policy effect. Although the two matching methods have good applicability in practice, potentially more suitable matching methods have not been tried. Third, the survey of CHARLS is only applicable for urban and rural residents over 45 years old. The utilisation of medical services by urban and rural residents younger than 45 years old cannot be considered, which limits the scope of application of this study. Finally, the investigation relied on time points, particularly 2011 and 2015. There may be a time lag effect on the policy effects that are not apparent in the short term and thus not considered in the analysis on the utilisation level of medical services.

\section{FURTHER RESEARCH}

The observed disparities in our study reveal the potential impact of the URRMI policy on the utilisation of medical services for urban and rural residents in China. However, the underlying mechanisms of the link between URRMI policy and the utilisation of medical services are complex. Further research is needed to explore potential mechanisms and validate this relationship in other methods. In addition, China has begun to implement the hierarchical treatment model in recent years and should pay attention to the rationality of medical service utilisation, including the rationality of admission and the rationality of the treatment.

\section{CONCLUSIONS}

The current policy design of URRMI has a significant impact on the medical service utilisation of rural residents, and it has positive affected the promotion of urban and rural equality in terms of outpatient utilisation. However, the current policy design has not yet obtained sufficient evidence tantamount to the promotion of hospitalisation equality between urban and rural residents. Policy makers should learn from the implementation of the pilot area and develop a much more effective implementation strategy for the upcoming national promotion of URRMI.

\section{Author affiliations}

'Department of Health Management, School of Medicine and Health Management, Tongji Medical College, Huazhong University of Science and Technology, Wuhan,

China

${ }^{2}$ Research Center for Rural Health Services, Hubei Province Key Research Institute of Humanities and Social Sciences, Wuhan, China

Acknowledgements The authors would like to thank the National Natural Science Foundation of China and the National School of Development, Peking University, and other members for their support and cooperation. We would also like to thank the study samples from China Health and Retirement Longitudinal Study (CHARLS) database for providing the information in our research.
Contributors DS, YC and HG contributed to the conception and design of the project; DS, HG, MT and ZC contributed to the analysis and interpretation of the data; HL, JC, DJ, XH and SL contributed to the data acquisition and provided statistical analysis support; DS drafted the article. All authors supplied critical revisions to the manuscript and gave final approval of the version to be published.

Funding This study was supported by grants from the National Natural Science Foundation of China (71673101 and 71473096).

Competing interests None declared.

Patient consent for publication Not required.

Ethics approval This study was approved by the Ethics Committee of the Tongji Medical College, Huazhong University of Science and Technology (IORG No: IORG0003571).

Provenance and peer review Not commissioned; externally peer reviewed. Data sharing statement No additional data are available.

Open access This is an open access article distributed in accordance with the Creative Commons Attribution Non Commercial (CC BY-NC 4.0) license, which permits others to distribute, remix, adapt, build upon this work non-commercially, and license their derivative works on different terms, provided the original work is properly cited, appropriate credit is given, any changes made indicated, and the use is non-commercial. See: http://creativecommons.org/licenses/by-nc/4.0/.

\section{REFERENCES}

1. World Health Organization. Sustainable health financing, universal coverage and social health insurance. World Health Assembly Resolution, 2005:58.

2. World Health Organization. World health report 2013: research for universal health coverage. World Health Assembly Resolution, 2013:7.

3. Basu S, Andrews J, Kishore S, et al. Comparative performance of private and public healthcare systems in low- and middle-income countries: a systematic review. PLoS Med 2012;9:e1001244.

4. Yip WC, Hsiao WC, Chen W, et al. Early appraisal of China's huge and complex health-care reforms. Lancet 2012;379:833-42.

5. Tang S, Brixi H, Bekedam $\mathrm{H}$. Advancing universal coverage of healthcare in China: translating political will into policy and practice. Int J Health Plann Manage 2014;29:160-74.

6. Li X, Zhang W. The impacts of health insurance on health care utilization among the older people in China. Soc Sci Med 2013;85:59-65.

7. Meng $Q, X u L$, Zhang $Y$, et al. Trends in access to health services and financial protection in China between 2003 and 2011: a crosssectional study. Lancet 2012;379:805-14.

8. National Health and Family Planning Commission of the People's Republic of China. 2017 China statistical yearbook of health and family planning. Beijing: Peking Union Medical College Press, 2017.

9. Department of Population and Employment Statistics of National Bureau of Statistics, Department of Planning and Finance of Ministry of Human Resource and Social Security. China Labour Statistical Yearbook 2015. Beijing: China Statistics Press, 2015.

10. Wang X, Zheng A, He X, et al. Integration of rural and urban healthcare insurance schemes in China: an empirical research. $B M C$ Health Serv Res 2014;14:142.

11. Lin X, Cai M, Tao H, et al. Insurance status, inhospital mortality and length of stay in hospitalised patients in Shanxi, China: a crosssectional study. BMJ Open 2017;7:e015884.

12. Zhu K, Zhang L, Yuan S, et al. Health financing and integration of urban and rural residents' basic medical insurance systems in China. Int J Equity Health 2017;16:194.

13. Meng $\mathrm{Q}$, Fang $\mathrm{H}$, Liu X, et al. Consolidating the social health insurance schemes in China: towards an equitable and efficient health system. Lancet 2015;386:1484-92.

14. Pan XF, Xu J, Meng Q. Integrating social health insurance systems in China. Lancet 2016;387:1274-5.

15. Eggleston K, Ling L, Qingyue M, et al. Health service delivery in China: a literature review. Health Econ 2008;17:149-65.

16. China's State Council. Opinion on integration of basic medical insurance systems between urban and rural residents (in Chinese). 2016 http://www.gov.cn/zhengce/content/2016-01/12/content 10582.htm (Accessed 20 Nov 2016).

17. Gu H, Ma C, Jj L. Decomposition of urban-rural differences in health care on arrangement area. Statistics and Information Forum 2013;6:89-94. 
18. Ma C, Zhao GC, Gu H. The effect of Urban-Rural Integrated Medical Insurance System on Rural Residents' Health Care Behavior. Statistical Research 2016;33:78-85.

19. Liu XL. The integrated urban and rural medical insurance and the utilization of medical care: evidence from China. The Journal of World Economy 2017;40:169-92.

20. Huang YF, Zhang JY. The impact of urban and rural medical insurance integration on utilization of rural residents' medical service: a case study on Guangzhou. Chinese Public Policy Review 2017.

21. Gebel M, Voßemer J. The impact of employment transitions on health in Germany. A difference-in-differences propensity score matching approach. Soc Sci Med 2014;108:128-36.

22. Sari N, Osman M. The effects of patient education programs on medication use among asthma and COPD patients: a propensity score matching with a difference-in-difference regression approach. BMC Health Serv Res 2015;15:332.

23. Zhang L, Wang Z, Qian D, et al. Effects of changes in health insurance reimbursement level on outpatient service utilization of rural diabetics: evidence from Jiangsu Province, China. BMC Health Serv Res 2014;14:185.

24. Andersen RM. Revisiting the behavioral model and access to medical care: does it matter? J Health Soc Behav 1995;36:1-10.

25. Nolan A. Evaluating the impact of eligibility for free care on the use of general practitioner (GP) services: a difference-in-difference matching approach. Soc Sci Med 2008;67:1164-72.

26. Cheng L, Liu H, Zhang Y, et al. The impact of health insurance on health outcomes and spending of the elderly: evidence from China's New Cooperative Medical Scheme. Health Econ 2015;24:672-91.

27. Lei X, Lin W. The New Cooperative Medical Scheme in rural China: does more coverage mean more service and better health? Health Econ 2009; 18:S25-46.

28. Hu Y, van Lenthe FJ, Hoffmann R, et al. Assessing the impact of natural policy experiments on socioeconomic inequalities in health: how to apply commonly used quantitative analytical methods? BMC Med Res Methodol 2017;17:17.

29. Baser O. Too much ado about propensity score models? Comparing methods of propensity score matching. Value Health 2006;9:377-85.

30. Ding L, Wu J. The impact of China's National Essential Medicine Policy and its implications for urban outpatients: a multivariate difference-in-differences study. Value Health 2017;20:20.

31. A z F, Dow WH, Liu GG, et al. Propensity score and difference-indifference methods: a study of second generation antidepressant use in patients with bipolar disorder[J]. Health Services \& Outcomes Research Methodology 2007;7:23-38.

32. Lagomarsino G, Garabrant A, Adyas A, et al. Moving towards universal health coverage: health insurance reforms in nine developing countries in Africa and Asia. Lancet 2012;380:933-43.

33. Long $\mathrm{Q}, \mathrm{Xu} \mathrm{L}$, Bekedam $\mathrm{H}$, et al. Changes in health expenditures in China in 2000s: has the health system reform improved affordability. Int $J$ Equity Health 2013;12:40.

34. Wang HQ, Liu ZH, Zhang YZ, et al. Integration of current identitybased district-varied health insurance schemes in China: implications and challenges. Front Med 2012;6:79-84.

35. Qiu P, Yang Y, Zhang J, et al. Rural-to-urban migration and its implication for new cooperative medical scheme coverage and utilization in China. BMC Public Health 2011;11:520.

36. Fang K, Shia B, Ma S, et al. Health insurance coverage and impact: a survey in three cities in China. PLoS One 2012;7:e39157.

37. Zhang $Y$, Tang W, Zhang X, et al. National Health Insurance Development in China from 2004 to 2011: coverage versus benefits. PLoS One 2015;10:e0124995.

38. Wang Q, Zhang D, Hou Z. Insurance coverage and socioeconomic differences in patient choice between private and public health care providers in China. Soc Sci Med 2016;170:124-32.

39. Chen W, Zhang Q, Renzaho AMN, et al. Social health insurance coverage and financial protection among rural-to-urban internal migrants in China: evidence from a nationally representative crosssectional study. BMJ Glob Health 2017;2:e000477.

40. Tang S, Meng Q, Chen L, et al. Tackling the challenges to health equity in China. Lancet 2008;372:1493-501.

41. You X, Kobayashi Y. The new cooperative medical scheme in China. Health Policy 2009;91:1-9.

42. Wagstaff $A$, Lindelow $M$, Jun $G$, et al. Extending health insurance to the rural population: an impact evaluation of China's new cooperative medical scheme. J Health Econ 2009;28:1-19.

43. Jian W, Chan KY, Reidpath DD, et al. China's Rural-urban care gap shrank for chronic disease patients, but inequities persist. Health Aff 2010;29:2189-96.

44. Fang $\mathrm{H}$, Meng $\mathrm{Q}$, Rizzo JA. Do different health insurance plans in China create disparities in health care utilization and expenditures? Int J Appl Econ 2014;11:1-18.

45. Mou J, Cheng J, Zhang D, et al. Health care utilisation amongst Shenzhen migrant workers: does being insured make a difference? BMC Health Serv Res 2009;9:214. 